\title{
Acreditação hospitalar como estratégia de melhoria: impactos em seis hospitais acreditados
}

\author{
Hospital accreditation as an improvement strategy: impacts and \\ difficulties in six accredited hospitals
}

\author{
Glauco Henrique de Sousa Mendes ${ }^{1}$ \\ Thayse Boucinha de Sousa Mirandola
}

\begin{abstract}
Resumo: A acreditação tem sido utilizada por muitos países para estimular a melhoria da qualidade dos serviços hospitalares. No Brasil, a difusão da acreditação hospitalar é de responsabilidade da Organização Nacional de Acreditação (ONA). Os estudos sobre os impactos da acreditação hospitalar têm despertado o interesse da comunidade acadêmica. O artigo tem o objetivo de analisar os impactos da acreditação no desempenho organizacional de hospitais. Foi adotada a abordagem qualitativa de pesquisa e o método de casos múltiplos. Foram realizados casos em seis hospitais acreditados do Estado de São Paulo. Os resultados evidenciaram que a acreditação hospitalar tem capacidade de gerar melhorias relacionadas à gestão dos processos, satisfação dos clientes e desenvolvimento de profissionais de saúde. O trabalho contribui ao ampliar os estudos sobre acreditação no Brasil

Palavras-chave: Acreditação hospitalar; Gestão da qualidade; Gestão hospitalar.
\end{abstract}

\begin{abstract}
Accreditation has been used by many countries to encourage improvements in hospital service quality. In Brazil, the dissemination of the Brazilian Accreditation System is a responsibility of the National Accreditation Organization (ONA). Studies on the impact of hospital accreditation have aroused the interest of the academic community. This article aims to analyze the impact of accreditation on organizational performance of hospitals. A qualitative research approach and the method of multiple cases were adopted for this study. Cases were performed in six accredited hospitals in the state of Sao Paulo. One of the main conclusions of this study is that hospital accreditation promotes improvements related to the process management, customer safety, and development of healthcare professionals. This paper contributes to expand the studies on accreditation in Brazil.
\end{abstract}

Keywords: Hospital accreditation; Quality management; Hospital management.

\section{Introdução}

A rede hospitalar no Brasil é diversificada em termos de infraestrutura, tipos de serviços e níveis de qualidade dos serviços prestados. Embora existam hospitais considerados de excelência, a grande maioria ainda precisa avançar em termos de qualidade dos serviços prestados (Burmester et al., 2007; Forgia \& Couttolenc, 2008).

Iniciativas de melhoria da qualidade nos serviços hospitalares são relatadas em diversos países (Malik \& Teles, 2001; Lee et al., 2002; Hasan \& Kerr, 2003; Manaf, 2005; Macinati, 2008; Alavi \& Mahamoud, 2008; Olástico \& Toledo, 2013). Uma das estratégias adotadas para melhorar a qualidade e a produtividade nas organizações de saúde é a criação de programas de acreditação hospitalar (Pomey et al., 2010).

Fortes et al. (2011) analisaram o sistema de acreditação em diferentes países e concluíram que não há um único modelo. As políticas nacionais e os incentivos dados para que os hospitais participem do processo de acreditação são variáveis que influenciam nos sistemas de acreditação. Em alguns países (por exemplo, no Reino Unido), a coordenação do sistema de acreditação tem sido delegada a organizações profissionais e o envolvimento dos hospitais é voluntário. Em outros países (França, por exemplo), percebe-se maior envolvimento do Estado como financiador da política de acreditação e a participação dos hospitais é compulsória (Pomey et al., 2005; Tabrizi et al., 2011; Fortes et al., 2011).

A acreditação hospitalar no Brasil ainda é recente. Ainda que as primeiras iniciativas de avaliação da qualidade dos serviços de saúde tenham sido feitas na década de setenta, foi só com a criação do Programa Brasileiro de Acreditação Hospitalar (PBAH) na década de noventa, que a acreditação ganhou maior projeção (Shiesari \& Kisil, 2003). O PBAH é operacionalizado

${ }^{1}$ Departamento de Engenharia de Produção, Universidade Federal de São Carlos - UFSCar, Rod.Washington Luís, Km 235, Caixa Postal 676, CEP 13565-905, São Carlos, SP, Brasil, e-mail: glauco@dep.ufscar.br; thaysemirandola@hotmail.com

Recebido em Jan. 29, 2014 - Aceito em Maio 20, 2015

Suporte financeiro: Nenhum. 
pela Organização Nacional de Acreditação (ONA), responsável pela difusão da acreditação entre as organizações de saúde, incluindo os hospitais. No Brasil, estima-se que menos que 5\% dos hospitais possuam o certificado de acreditação (ONA, 2013). É um número tímido em relação ao total de hospitais existentes no País.

Dentre os potenciais impactos da acreditação estariam a melhoria do gerenciamento da organização hospitalar e a da qualidade da assistência prestada ao usuário (Chen et al., 2003; El-Jardali et al., 2008; Figueiredo, 2012). Além disso, a acreditação pode garantir reconhecimento público ao hospital acreditado (Feldman et al., 2005). Ainda que tais resultados possam ser alcançados, estudos sobre acreditação hospitalar revelam um quadro complexo e apontam para a necessidade de mais pesquisas para se determinar os reais impactos da acreditação (Greenfield \& Braithwaite, 2008; Pomey et al., 2010; Hinchcliff et al., 2012)

Este artigo tem o objetivo de descrever e analisar os impactos da acreditação em hospitais paulistas. O termo impacto deve ser entendido como os efeitos do processo de acreditação na gestão hospitalar, possibilitando as mudanças organizacionais, mudanças no envolvimento dos profissionais de saúde e na melhoria dos serviços prestados.

A pesquisa foi conduzida em seis hospitais acreditados, todos localizados no Estado de São Paulo. O método utilizado foi o estudo de casos múltiplos (lógica da replicação), já que este método é adequado quando o objetivo é expor as condições de um determinado fenômeno (Yin, 2001). Para Eisenhardt (1989), o estudo de caso é um método importante adotado na área de gestão como forma de análise de dados qualitativos e para construção de novas teorias.

\section{Revisão teórica}

\subsection{Acreditação hospitalar}

A acreditação hospitalar surgiu nos Estados Unidos. Sua origem está relacionada a uma iniciativa do Colégio Americano de Cirurgiões, que em 1924 criou o Programa de Padronização Hospitalar. O objetivo foi estabelecer um conjunto de padrões para garantir a qualidade da assistência aos pacientes. Em 1950, o número de avaliações já superava os três mil hospitais (Feldman et al., 2005).

Na década de cinquenta, o programa de acreditação americano foi delegado a Joint Commission on Accreditation of Hospitals (nomeada posteriormente de Joint Commission), uma organização de natureza privada. Na década seguinte, como a maioria dos hospitais americanos já havia atingido os padrões mínimos de qualidade, a Joint Commission elevou o grau de exigência. Em 1970, publicou o Accreditation
Manual for Hospital, contendo padrões ótimos de qualidade e considerando também processos e resultados da assistência nas avaliações (Feldman et al., 2005).

A partir dos anos oitentas, os programas de acreditação se expandiram para outros países. Inicialmente, nos países de língua inglesa (Canadá e Austrália) e Europa. Na década seguinte, para países da América Latina e Ásia. No caso brasileiro, a acreditação começou efetivamente no final dos anos oitenta, sob a influência da Organização Pan-Americana da Saúde (OPAS), que estabeleceu uma série de padrões para os serviços hospitalares da América Latina (Shiesari \& Kisil, 2003).

Em 1997, o Ministério da Saúde criou uma comissão nacional de especialistas para desenvolver o modelo brasileiro de acreditação. Um dos seus resultados foi a publicação do Manual Brasileiro de Acreditação Hospitalar, que estabelecia padrões de qualidade e segurança para os hospitais que buscavam a acreditação. Em maio de 1999, foi criada a Organização Nacional de Acreditação (ONA), uma entidade não governamental e sem fins lucrativos com a atribuição de coordenar o Sistema Brasileiro de Acreditação por meio da implantação de padrões e normas técnicas, credenciamento de instituições acreditadoras e capacitação de avaliadores (Shiesari \& Kisil, 2003).

A ONA tem o objetivo de promover a implantação da certificação da qualidade dos serviços de saúde em diferentes categorias de organizações de saúde, que voluntariamente optam por aderir à acreditação (ONA, 2013). No artigo, será considerado apenas o processo de acreditação hospitalar.

No caso brasileiro, a acreditação consiste num processo de avaliação voluntário, periódico e reservado, que tende a garantir a qualidade da assistência por meio de padrões previamente aceitos. Tais padrões são estabelecidos em grau de complexidade crescente e servem de guia para que uma instituição avaliadora possa avaliar a infraestrutura, os processos e os resultados de um hospital, dependendo do nível de acreditação a ser implantada (ONA, 2013).

Segundo o modelo brasileiro, um hospital pode ser certificado em três diferentes níveis de complexidade (ONA, 2013):

- Nível 1 (Acreditação): caracterizado pela ênfase na segurança. Verifica o atendimento de requisitos formais, técnicos e de estrutura conforme a legislação correspondente. Enfatiza também a gestão de riscos adotada pelo hospital.

- Nível 2 (Acreditação Plena): tem foco nos processos. Avalia a gerência dos processos e suas interações sistêmicas. Também pressupõe um sistema de medição de desempenho e ações de educação para melhoria desses processos. 
- Nível 3 (Acreditação com Excelência): tem foco nos resultados. Avalia a existência de políticas de melhoria contínua do desempenho organizacional a partir das medições internas e comparações externas. Neste nível, há evidências de maior maturidade na gestão hospitalar e uso estratégico das ações de melhoria.

Como o processo de acreditação nacional é de caráter voluntário, cabe ao hospital a prerrogativa de analisar os potenciais benefícios gerados pela acreditação e decidir pela sua implantação ou não (ONA, 2013).

\subsection{Impactos da acreditação}

A identificação dos impactos da acreditação revela um quadro complexo e, por sua vez, com resultados contraditórios (Ovretveit \& Gustafson, 2002; Greenfield \& Braithwaite, 2008; Pomey et al., 2010).

A Figura 1 apresenta uma síntese dos principais impactos apontados na bibliografia e abordados neste artigo. Os impactos foram agrupados em três categorias: a) mudanças organizacionais e nas práticas hospitalares; b) impactos no comportamento dos profissionais de saúde; e c) impactos na satisfação de pacientes e no reconhecimento público.

\subsubsection{Mudanças organizacionais e nas práticas hospitalares}

Parece haver consenso de que a acreditação atua como promotora de mudanças organizacionais (Greenfield \& Braithwaite, 2008). Mudanças na gestão dos processos, nas práticas de segurança e nos comportamentos das pessoas são algumas das principais mudanças apontadas na bibliografia (Pomey et al., 2004). A acreditação também contribui para a criação de uma cultura voltada para a qualidade e segurança aos pacientes (ElJardali et al., 2008; Pomey et al., 2010).

Mais especificamente, estudos buscaram avaliar os impactos da acreditação nas práticas clínicas. Sekimoto et al. (2008) sugeriram que a acreditação afeta positivamente a introdução de programas de controle de infecção em hospitais japoneses. Devers et al. (2004) identificaram uma redução no número de erros médicos em hospitais americanos acreditados pela Joint Commission. Apesar de esses estudos apresentarem evidências positivas, resultados divergentes também têm sido encontrados (Miller et al., 2005). Neste sentido, Greenfield et al. (2012b) apontam que há falta de evidências empíricas robustas para se estabelecer uma relação definitiva entre a acreditação e a melhoria das práticas clínicas.

A adequação aos padrões de qualidade preconizados pela acreditação faz com que o hospital tenha que rever seus processos e métodos de trabalho. Alkhenizan \& Shaw (2011) concluíram que os programas de acreditação melhoram os processos hospitalares e, portanto, devem ser vistos como uma importante ferramenta de qualidade. Shaw et al. (2010) encontraram evidências de que hospitais acreditados ou aqueles que possuíam certificação ISO 9001 apresentavam melhores desempenhos em termos de gestão, controle de riscos e satisfação de pacientes em comparação com hospitais sem nenhuma certificação. Entretanto, todo este esforço de sistematização pode gerar uma carga significativa de trabalho em termos de tarefas administrativas, o que pode se tornar uma disfunção e fonte de resistência à acreditação (Pomey et al., 2004).

Existe a percepção de que a implantação da acreditação também traz melhorias nos indicadores de qualidade e de desempenho. Entretanto, a relação entre os indicadores de qualidade e acreditação não é consistente, já que pesquisas mostram não haver correlação entre essas duas variáveis (Miller et al., 2005).

Por fim, a avaliação do impacto financeiro da acreditação ainda é um ponto obscuro. Há dificuldades para se mensurar economicamente os resultados alcançados, já que melhorias na assistência aos pacientes e de eficiência nos processos nem sempre podem ser associadas diretamente à acreditação. Há a necessidade de mais estudos sobre a avaliação econômica da acreditação (Greenfield \& Braithwaite, 2008; Mumford et al., 2013).

\subsubsection{Impactos no comportamento dos profissionais de saúde}

Para Pomey et al. (2005), a implantação da acreditação hospitalar necessita do envolvimento de diversos segmentos profissionais (alta direção, médicos, enfermeiros e pessoal administrativos). Para Manzo et al. (2012), a acreditação é influenciada pelo nível de motivação dos trabalhadores, ao mesmo tempo que ela influencia o ambiente de trabalho desses profissionais.

Engajamentos e resistências dos profissionais de saúde em relação à acreditação têm sido observados. A alta direção é responsável por estabelecer o propósito da organização e, no caso da acreditação, seu engajamento serve como exemplo para os demais profissionais de saúde (Macinati, 2008; El-Jardali et al., 2008). $\mathrm{O}$ envolvimento dos profissionais de enfermagem é apontado como relevante nos processos de acreditação. Este profissional, normalmente, assume funções técnicas e de gestão, desempenhando uma posição-chave na acreditação (Manzo et al., 2012). Por sua vez, a relutância do corpo médico em se envolver ativamente nos processos de acreditação tem sido abordada por diferentes pesquisas (Øvretveit, 1996; Mezomo, 2001; Manzo, 2009). 
Um ponto de consenso em relação aos impactos da acreditação hospitalar é que ela contribui para o aumento da qualificação dos profissionais de saúde (Greenfield \& Braithwaite, 2008; Greenfield et al., 2011). A acreditação estimula a realização de atividades de treinamento e educação, o que contribui para a capacitação dos profissionais de saúde e o aprendizado organizacional (Pomey et al., 2005; Newhouse, 2006; Touati \& Pomey, 2009).

Quanto às atitudes e percepções que os profissionais de saúde têm em relação à acreditação, são observadas posições contrastantes. Quando se consideram os clientes internos, há pesquisas que revelam que a acreditação contribui para o aumento da satisfação dos profissionais de saúde (Al Tehewy et al., 2009). As visões positivas associam a acreditação como uma estratégia eficaz para a qualidade e melhoria do desempenho organizacional (Davis et al., 2007; Alkhenizan \& Shaw, 2011). Também, seria uma forma de demonstrar compromisso com a qualidade dos serviços (Devers et al., 2004; Auras \& Geraedts, 2010). Já as atitudes negativas associam a acreditação à burocratização e aos seus altos custos de implantação (Pomey et al., 2004).

\subsubsection{Impactos na satisfação de pacientes e reconhecimento público}

A capacidade da acreditação em gerar melhorias na satisfação dos pacientes e conceder reconhecimento público aos hospitais acreditados são argumentos importantes em seu favor (Al Tehewy et al., 2009; Thornlow \& Merwin, 2009). Assim, a acreditação deve ser entendida também como uma estratégia de marketing que transmite valor à marca do hospital, diferenciando-o da concorrência.

Entretanto, estudos (Greenfield \& Braithwaite, 2008; Hinchcliff et al., 2012) demonstraram não haver uma correlação consistente entre a acreditação e a satisfação dos pacientes. Pesquisa realizada por Sack et al. (2011) com 37.000 pacientes em 78 hospitais revelou que a acreditação não é associada diretamente à melhoria da qualidade de assistência. Os autores concluem que a acreditação é um passo importante na gestão da qualidade, mas não é diretamente percebida pelos pacientes. Portanto, este é um tema que ainda demanda maiores investigações.

A melhoria da imagem do hospital seria um impacto da acreditação (El-Jardali et al., 2008). Ainda que a divulgação dos relatórios de avaliação não seja consensual (há modelos com total transparência e outros confidenciais), a divulgação dos resultados finais, seja total ou parcial, é considerada positiva para pacientes e para o próprio hospital (Greenfield \& Braithwaite, 2008).

Greenfield \& Braithwaite (2008) destacaram que, dos impactos apresentados na Figura 1, apenas a capacidade de promover mudanças e de estimular o desenvolvimento dos profissionais de saúde foram efetivamente comprados. Os demais impactos ainda necessitam de mais estudos, já que há os resultados encontrados até o momento se mostraram inconsistentes.

\section{Método de pesquisa}

Foi adotada a abordagem qualitativa de pesquisa e o método de casos múltiplos. A pesquisa tem natureza qualitativa e descritiva, o que também conduz à estratégia do estudo de caso devido à natureza dos dados nesse tipo de abordagem. A análise dos dados seguiu o método indutivo, no qual, a partir de constatações particulares, pode-se chegar a conclusões genéricas, ainda que respeitadas as limitações deste tipo estudo.

Para Yin (2001), o estudo de caso é uma estratégia de pesquisa empírica que investiga um fenômeno dentro do contexto da vida real. A escolha dessa abordagem foi justificada em função da necessidade de se compreender um fenômeno social complexo e cujo contexto é relevante, pois se busca analisar os impactos da acreditação em hospitais paulistas. Ainda segundo o autor, o método de casos múltiplos segue a mesma estrutura metodológica do estudo de caso e deve ser utilizado quando se buscam replicações literais ou teóricas sobre um determinado contexto. No artigo, seguiu-se a lógica das replicações literais (Yin, 2001).

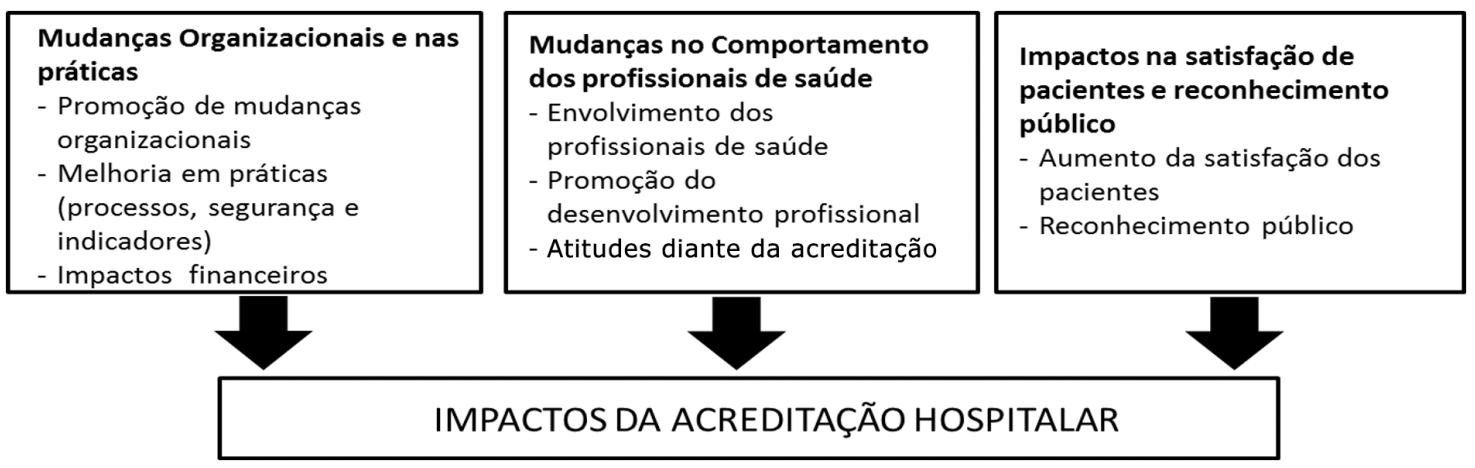

Figura 1. Síntese dos principais impactos da acreditação. Fonte: elaborado pelos autores. 
Na primeira etapa da pesquisa, foi realizado um levantamento bibliográfico sobre o tema acreditação hospitalar. Depois de sua conclusão, foi elaborado um protocolo a fim de garantir a homogeneidade nas práticas de investigação.

Os hospitais escolhidos como objeto de estudo atenderam aos seguintes requisitos da pesquisa: a) serem acreditados e b) estarem disponíveis para a pesquisa. Portanto, a seleção foi intencional e os hospitais foram identificados por meio de uma lista de hospitais acreditados disponibilizada pela ONA (ONA, 2013). Seis hospitais foram selecionados como unidades de análise, sendo todos acreditados. Optou-se por buscar hospitais com diferentes níveis de acreditação. Os hospitais participantes não autorizaram a divulgação de seus nomes e, consequentemente, foi utilizada outra denominação para identificá-los.

Todos os hospitais possuem uma área funcional dedicada à gestão da qualidade e que também é responsável pela coordenação da acreditação. As informações foram coletadas por meio de entrevistas semiestruturadas com os responsáveis pela implantação da acreditação, que, por sua vez, também respondiam pela gestão da qualidade nesses hospitais. Os dados dos hospitais participantes e dos entrevistados são mostrados no Quadro 1. Os estudos de caso foram conduzidos entre maio e junho de 2013 e as entrevistas tiveram duração média de 2 a 3 horas.

$\mathrm{O}$ roteiro de entrevista contemplou questões relativas à caracterização do hospital, ao histórico da acreditação (motivações, apoios externos, organização interna etc.), principais ações implantadas e identificação dos benefícios e das dificuldades da acreditação. As questões eram, em sua maioria, de natureza aberta, possibilitando a troca de informações entre o respondente e o pesquisador. Neste mesmo roteiro, entretanto, havia um conjunto de questões de natureza fechada, no qual se pedia aos entrevistados que avaliassem os impactos da acreditação. Para tanto, foi utilizada uma escala Likert de 1 a 5 (representando o grau de concordância em relação aos impactos). Apesar do uso desta estratégia de coleta dos dados, o estudo mantém sua natureza qualitativa.

Para maior confiabilidade da pesquisa, buscou-se a triangulação dos dados por meio da utilização de diferentes fontes de evidências (Yin, 2001). Além das entrevistas, foram utilizadas outras fontes complementares, tais como documentos fornecidos pelos entrevistados e observação direta, que foi realizada de maneira informal nas visitas aos hospitais.

Buscou-se interpretar as entrevistas por meio da análise de conteúdo. O objetivo era extrair informações dadas pelos entrevistados que pudessem ser categorizadas em dois temas: benefícios e dificuldades da acreditação. A técnica foi realizada em três etapas: leitura inicial, exploração do material e tratamento dos resultados (Bardin, 1977).

\section{Estudo de casos}

\subsection{Caracterização dos hospitais da pesquisa}

Os casos não serão descritos isoladamente, mas de forma conjunta, o que permite uma melhor comparação entre os hospitais pesquisados. As principais características dos hospitais estão sintetizadas no Quadro 1.

Quanto ao porte, os hospitais H1, H2 e H5 são considerados de médio porte, pois possuem entre 50 e 149 leitos. Os hospitais H3, H4 e H6 são considerados hospitais de grande porte por possuírem mais de 150 leitos. Todos os hospitais são

Quadro 1. Caracterização dos hospitais pesquisados.

\begin{tabular}{|c|c|c|c|c|c|c|}
\hline \multirow{2}{*}{ Características } & \multicolumn{6}{|c|}{ HOSPITAIS } \\
\hline & H1 & H2 & H3 & H4 & H5 & H6 \\
\hline Porte & Médio & Médio & Grande & Grande & Médio & Grande \\
\hline Tipo & Geral & Geral & Geral & Geral & Geral & Geral \\
\hline Natureza & Privado & Cooperativa & Público & Público & Privado & Privado \\
\hline Colaboradores & 523 & 350 & 1.459 & 1.579 & 474 & 834 \\
\hline $\begin{array}{l}\text { Atendimento } \\
\text { Leitos Totais }\end{array}$ & $\begin{array}{l}90 \text { leitos } \\
10 \text { UTI }\end{array}$ & $\begin{array}{l}56 \text { leitos } \\
10 \text { UTI }\end{array}$ & $\begin{array}{l}243 \text { leitos } \\
38 \text { UTI }\end{array}$ & $\begin{array}{l}319 \text { leitos } \\
20 \text { UTI }\end{array}$ & $\begin{array}{l}87 \text { leitos } \\
22 \text { UTI }\end{array}$ & $\begin{array}{l}201 \text { leitos } \\
28 \text { UTI }\end{array}$ \\
\hline Primeira Acreditação & $\begin{array}{c}\text { Acreditado } \\
(2005)\end{array}$ & $\begin{array}{c}\text { Acreditado } \\
\text { (2012) }\end{array}$ & $\begin{array}{c}\text { Acreditado } \\
(2011)\end{array}$ & $\begin{array}{c}\text { Acreditado } \\
(2004)\end{array}$ & $\begin{array}{c}\text { Acreditado } \\
(2001)\end{array}$ & $\begin{array}{c}\text { Acreditado } \\
(2010)\end{array}$ \\
\hline $\begin{array}{l}\text { Acreditação } \\
\text { atual }\end{array}$ & $\begin{array}{l}\text { Acreditado } \\
\text { com } \\
\text { excelência } \\
(2013)\end{array}$ & $\begin{array}{c}\text { Acreditado } \\
\text { (2012) }\end{array}$ & $\begin{array}{c}\text { Acreditado } \\
\text { Pleno } \\
\text { (2013) }\end{array}$ & $\begin{array}{c}\text { Acreditado } \\
\text { (2012) }\end{array}$ & $\begin{array}{l}\text { Acreditado } \\
\text { com } \\
\text { excelência } \\
\text { (2012) }\end{array}$ & $\begin{array}{l}\text { Acreditado } \\
\text { Pleno } \\
(2012)\end{array}$ \\
\hline $\begin{array}{l}\text { Apoio de consultoria } \\
\text { externa }\end{array}$ & Sim & Sim & Sim & Sim & Sim & Não \\
\hline Cargo do Entrevistado & $\begin{array}{l}\text { Consultora } \\
\text { Interna da } \\
\text { Qualidade }\end{array}$ & $\begin{array}{l}\text { Supervisora } \\
\text { de } \\
\text { Enfermagem }\end{array}$ & $\begin{array}{l}\text { Gerente da } \\
\text { qualidade }\end{array}$ & $\begin{array}{l}\text { Coordenadora } \\
\text { da Qualidade }\end{array}$ & $\begin{array}{l}\text { Gerente da } \\
\text { Qualidade }\end{array}$ & $\begin{array}{l}\text { Coordenador } \\
\text { da Qualidade }\end{array}$ \\
\hline
\end{tabular}


caracterizados como hospitais gerais, ou seja, não são dedicados a uma especialidade médica. Quanto à natureza jurídica, os hospitais $\mathrm{H} 3$ e H4 são públicos, os hospitais H1, H5 e H6 são privados e o Hospital H2 é uma cooperativa.

Foram pesquisados hospitais nos três níveis de acreditação. Os hospitais H2 e H4 são acreditados (nível 1), os hospitais H3 e H6 possuem a certificação de acreditação plena (nível 2) e os hospitais H1 e H5 têm a certificação mais elevada, que é a de acreditação com excelência (nível 3). Entre os hospitais pesquisados, cinco passaram por processos de recertificação, dos quais quatro elevaram o nível de certificação (H1, H3, H5 e H6). As decisões de recertificação e de elevação do nível da acreditação denotam o compromisso dos hospitais com a melhoria da gestão e dos serviços prestados.

Para a implantação da acreditação, cinco hospitais (H1, H2, H3, H4 e H5) contaram como o apoio de consultorias externas, que se envolveram nas etapas de diagnóstico, treinamento, mapeamento de processos, criação de indicadores e realização de auditorias internas. No caso do Hospital H6, a implantação ocorreu sem a participação de consultoria externa. Apesar do apoio das consultorias, os entrevistados ressaltaram que a acreditação só se tornou viável com a formação de equipes internas para condução do processo de implantação.

Houve convergência nos motivos que levaram os hospitais pesquisados a buscarem a acreditação.
Todos afirmaram que acreditação consiste numa metodologia para se alcançar a qualidade da assistência e segurança aos usuários. Neste caso, há uma nítida associação da acreditação hospitalar como um meio para avaliar, implantar e melhorar as práticas de qualidade nos hospitais. O segundo motivo apontado foi apontar a acreditação como uma forma de gestão, principalmente focada na padronização e melhoria dos processos. Apenas o hospital $\mathrm{H} 2$ apontou a necessidade de reconhecimento externo como um fator motivador para a sua certificação.

\subsection{Mudanças implantadas}

Os padrões da acreditação estão baseados em três dimensões: estrutura, processos e resultado. Foi solicitado aos entrevistados que destacassem as principais mudanças implantadas nessas dimensões. O Quadro 2 apresenta uma síntese das respostas.

Na dimensão estrutura (Nível 1), o foco é a garantia de existência de recursos técnicos e de infraestrutura necessários à prestação dos serviços hospitalares. A pesquisa enfatizou a necessidade de mudanças na infraestrutura física dos hospitais pesquisados. Conforme mostra o Quadro 2, quatro hospitais tiveram que promover mudanças em suas instalações físicas. O Hospital $\mathrm{H} 1$ adequou seu pronto-atendimento (PA), ampliando a sala de espera, consultórios clínicos e salas para pequenos procedimentos cirúrgicos. O Hospital H3 também precisou fazer melhorias

Quadro 2. Mudanças implantadas devido à acreditação.

\begin{tabular}{|c|c|c|c|c|}
\hline \multirow[b]{2}{*}{ HOSPTIAIS } & \multicolumn{4}{|c|}{ DIMENSÕES DA ACREDITAÇÃO } \\
\hline & $\begin{array}{c}\text { Estrutura - } \\
\text { Infraestrutura }\end{array}$ & $\begin{array}{c}\text { Estrutura - } \\
\text { Gerenciamento do } \\
\text { Risco }\end{array}$ & Processos & Resultados \\
\hline H1 & $\begin{array}{l}\text { Ampliação do pronto } \\
\text { atendimento }\end{array}$ & $\begin{array}{l}\text { Implantação de } \\
\text { barreiras preventivas }\end{array}$ & $\begin{array}{l}\text { Criação de protocolos } \\
\text { assistenciais e } \\
\text { diretrizes clínicas }\end{array}$ & $\begin{array}{l}\text { Acompanhamento } \\
\text { dos resultados dos } \\
\text { processos }\end{array}$ \\
\hline $\mathbf{H 2}$ & Não houve & $\begin{array}{l}\text { Ênfase nos processos } \\
\text { para notificações e } \\
\text { análise de eventos } \\
\text { adversos }\end{array}$ & $\begin{array}{l}\text { Implantação de } \\
\text { protocolos }\end{array}$ & $\begin{array}{l}\text { Ênfase na implantação } \\
\text { de melhorias da } \\
\text { instituição }\end{array}$ \\
\hline H3 & $\begin{array}{l}\text { Adequação de áreas } \\
\text { para pacientes e postos } \\
\text { de enfermagem }\end{array}$ & $\begin{array}{l}\text { Estímulo à cultura } \\
\text { de gerenciamento de } \\
\text { risco, que não estava } \\
\text { disseminada }\end{array}$ & $\begin{array}{l}\text { Documentação } \\
\text { física dos padrões e } \\
\text { capacitação }\end{array}$ & $\begin{array}{l}\text { Implantado o sistema } \\
\text { de gestão estratégica }\end{array}$ \\
\hline H4 & Não houve & $\begin{array}{l}\text { Programa de gestão } \\
\text { de risco já estava } \\
\text { implantado, mas houve } \\
\text { reforço das ações }\end{array}$ & $\begin{array}{l}\text { Ênfase na gestão de } \\
\text { processos }\end{array}$ & $\begin{array}{l}\text { Constantes mudanças } \\
\text { de gestores }\end{array}$ \\
\hline H5 & $\begin{array}{l}\text { Ampla reforma e } \\
\text { ampliação dos espaços } \\
\text { internos }\end{array}$ & $\begin{array}{l}\text { Criação do comitê de } \\
\text { gerenciamento } \\
\text { de risco }\end{array}$ & $\begin{array}{l}\text { Descrição de } \\
\text { Todos os processos e } \\
\text { procedimentos internos }\end{array}$ & $\begin{array}{l}\text { Planejamento } \\
\text { estratégico baseado em } \\
\text { resultados operacionais }\end{array}$ \\
\hline H6 & $\begin{array}{l}\text { Adequação dos setores } \\
\text { do almoxarifado e } \\
\text { farmácia }\end{array}$ & $\begin{array}{l}\text { Identificação do risco } \\
\text { nos pacientes e normas } \\
\text { para notificação de } \\
\text { eventos adversos }\end{array}$ & $\begin{array}{l}\text { Enfase em protocolos } \\
\text { multidisciplinares }\end{array}$ & $\begin{array}{l}\text { Gestão por indicadores } \\
\text { e planejamento } \\
\text { estratégico da } \\
\text { instituição }\end{array}$ \\
\hline
\end{tabular}


em suas instalações, principalmente quanto aos quartos dos pacientes e postos de enfermagem. Para o hospital $\mathrm{H} 5$, a mudança foi maior e mais dispendiosa (investimento em equipamentos, centro cirúrgico e área de conforto médico), porém necessária para reforçar o posicionamento de qualidade superior adotado pelo hospital. No hospital H6, as áreas de Almoxarifado e Farmácia passaram por mudanças físicas.

Outra questão importante nesta dimensão é o gerenciamento de risco. No Hospital H1 foram implantadas barreiras preventivas aos riscos identificados. No Hospital H2 houve mudanças nos processos para notificação e análise de eventos adversos. Para o Hospital H3, foram tomadas ações de sensibilização do corpo clínico a fim de criar uma cultura de gerenciamento de risco. No Hospital H4, já havia um programa de gerenciamento de risco implantado há dois anos e a acreditação serviu para reforçar sua importância. No Hospital H5, foi criado um comitê de gerenciamento de risco. Por fim, o hospital H6 adotou ações para classificação do risco no atendimento de urgência e criação de normas para notificação de eventos.

$\mathrm{Na}$ dimensão processo, os hospitais devem possuir processos mapeados, padronizados e devidamente documentados. Todos os seis hospitais, independentemente do nível de acreditação, afirmaram ter investido em técnicas de gestão de processos. Por exemplo, no Hospital H3 houve um grande esforço da gerência da qualidade a fim de implantar toda a documentação física relativa aos protocolos hospitalares, prática que era não estruturada antes da acreditação. Para o Hospital H4, muitas mudanças ocorreram nesta dimensão, atualmente tudo é baseado em normas técnicas e protocolos documentados. Já para o Hospital H6, houve ênfase na integração das áreas por meio de protocolos multidisciplinares e procedimentos interligados.

$\mathrm{Na}$ dimensão resultados, o foco recai sobre a qualidade da gestão do hospital e sua capacidade de gerar políticas de melhoria dos resultados. A maioria dos entrevistados concorda que a acreditação propiciou melhorias na gestão dos hospitais. Os entrevistados dos hospitais $\mathrm{H} 1$ e $\mathrm{H} 2$ destacaram o aumento da organização interna em função da normatização, do gerenciamento e a melhoria dos processos. $\mathrm{O}$ uso de indicadores de desempenho e a implantação de processos de planejamento estratégico foram ressaltados pelos respondentes dos hospitais $\mathrm{H} 3, \mathrm{H} 5$ e H6. Entretanto, para a coordenadora da qualidade do hospital H4, mudanças sucessivas na direção do hospital comprometeram os resultados nessa dimensão.

\subsection{Impactos da acreditação}

Foi solicitado aos entrevistados que assinalassem, em uma escala de 1 (discordo totalmente) a 5 (concordo totalmente), a opção que melhor representava a situação de seu hospital em relação aos impactos da acreditação (Quadros 3, 4 e 5). Posteriormente, argumentos deveriam ser dados para justificar o grau de concordância apontado. Os resultados são apresentados nas próximas seções.

Quadro 3. Impactos da acreditação - Mudanças organizacionais e nas práticas hospitalares.

\begin{tabular}{|l|c|c|c|c|c|c|c|c|}
\hline \multicolumn{1}{|c|}{ Impactos - organizacionais e práticas hospitalares } & H1 & H2 & H3 & H4 & H5 & H6 & Média & $\begin{array}{c}\text { Desvio } \\
\text { padrão }\end{array}$ \\
\hline Capacidade de promover mudanças na gestão hospitalar & 5 & 4 & 5 & 4 & 5 & 4 & $\mathbf{4 , 5 0}$ & $\mathbf{0 , 5 5}$ \\
\hline Mudanças na tomada de decisão & 4 & 4 & 4 & 4 & 5 & 4 & $\mathbf{4 , 1 7}$ & $\mathbf{0 , 4 1}$ \\
\hline Melhorias na qualidade dos serviços prestados & 5 & 5 & 5 & 5 & 5 & 4 & $\mathbf{4 , 8 3}$ & $\mathbf{0 , 4 1}$ \\
\hline Segurança e diminuição de riscos aos pacientes & 5 & 4 & 5 & 5 & 5 & 4 & $\mathbf{4 , 6 7}$ & $\mathbf{0 , 5 2}$ \\
\hline Mudanças nas rotinas e procedimentos & 5 & 4 & 4 & 5 & 5 & 4 & $\mathbf{4 , 5 0}$ & $\mathbf{0 , 5 5}$ \\
\hline $\begin{array}{l}\text { Compromisso com os processos de avaliação da } \\
\text { qualidade }\end{array}$ & 4 & 4 & 5 & 4 & 5 & 4 & $\mathbf{4 , 3 3}$ & $\mathbf{0 , 5 2}$ \\
\hline Melhoria nos indicadores de qualidade & 5 & 4 & 5 & 4 & 5 & 4 & $\mathbf{4 , 5 0}$ & $\mathbf{0 , 5 5}$ \\
\hline Investimento financeiro versus benefícios gerados & 4 & 3 & 4 & 3 & 3 & 3 & $\mathbf{3 , 3 3}$ & $\mathbf{0 , 5 1}$ \\
\hline
\end{tabular}

Quadro 4 . Impactos da acreditação - Impactos no comportamento dos profissionais de saúde.

\begin{tabular}{|l|c|c|c|c|c|c|c|c|}
\hline Impactos - comportamento dos profissionais de saúde & H1 & H2 & H3 & H4 & H5 & H6 & Média & $\begin{array}{c}\text { Desvio } \\
\text { padrão }\end{array}$ \\
\hline Desenvolvimento do pessoal de enfermagem & 4 & 5 & 5 & 4 & 5 & 4 & $\mathbf{4 , 5 0}$ & $\mathbf{0 , 5 5}$ \\
\hline Desenvolvimento do pessoal médico & 4 & 4 & 5 & 2 & 3 & 3 & $\mathbf{3 , 5 0}$ & $\mathbf{1 , 0 5}$ \\
\hline Desenvolvimento do pessoal da administração & 4 & 4 & 5 & 4 & 4 & 3 & $\mathbf{4 , 0 0}$ & $\mathbf{0 , 6 3}$ \\
\hline Mudança positiva na atitude do profissional & 5 & 5 & 4 & 4 & 5 & 3 & $\mathbf{4 , 3 3}$ & $\mathbf{0 , 8 2}$ \\
\hline Mudança negativa na atitude do profissional & 1 & 2 & 2 & 2 & 1 & 2 & $\mathbf{1 , 6 6}$ & $\mathbf{0 , 5 1}$ \\
\hline
\end{tabular}




\subsubsection{Mudanças organizacionais e nas práticas hospitalares}

O Quadro 3 apresenta a percepção dos entrevistados quanto aos impactos relativos à categoria mudanças organizacionais e mudanças nas práticas hospitalares.

Os entrevistados corroboraram a afirmação de que a acreditação é capaz de promover mudanças na gestão dos hospitais. Foram identificadas mudanças nos processos de tomada de decisão, que passaram a ser baseados em fatos e dados. Do mesmo modo, a acreditação estimulou o compromisso do hospital com os processos de avaliação da qualidade. Os trechos abaixo ilustram a relação entre acreditação e geração de mudanças organizacionais:

[...] desenvolvemos um método de acompanhamento do desempenho de cada processo. Através desses indicadores é possivel propor melhorias após análise de causas. (Hospital H1)

[...] hoje nos preocupamos muito com a comunicação, algo que não é fácil de se implantar, são diversos meios de comunicação e para diferentes públicos. (Hospital H2)

[...] Realizamos mensalmente a análise crítica dos indicadores com acompanhamento estratégico e tomada de decisão pela alta administração e lideranças dos setores do hospital.(Hospital H3)

Um ponto de destaque refere-se à relação entre a acreditação e a melhoria dos processos. Há uma alta percepção de que a acreditação é capaz de melhorar os processos de trabalho, o que contribui para diminuição dos riscos e aumento da segurança aos pacientes. Deste modo, a acreditação é um elemento importante para que o hospital estabeleça seu sistema de gestão da qualidade, já que estimula a melhor gestão dos processos e organização interna, o que pode ser evidenciado nos trechos:

[...] a acreditação traz a padronização dos processos, com isto tivemos melhorias principalmente nos serviços das áreas de apoio (laboratórios, diagnóstico por imagem etc.). (Hospital H4)

[...] ela (acreditação) nos deu um entendimento daquilo que fazemos e como os setores estão relacionados. Fizemos um grande esforço para padronizar nossas atividades. (Hospital H6)

Quanto ao retorno financeiro da acreditação, os entrevistados optaram por respostas neutras, o que resultou numa média de 3,33. Isto denota a dificuldade de se mensurar economicamente os ganhos advindos com a acreditação. Metodologias para mensuração dos custos da não qualidade poderiam ser empregadas para se comparar retornos financeiros e custos com a acreditação.

\subsubsection{Impactos no comportamento dos profissionais de saúde}

Foram identificados diferentes graus de envolvimentos dos profissionais de saúde com a acreditação. Como mostrado no Quadro 6 o envolvimento da alta direção foi considerado alto em todos os hospitais. Como em toda estratégia de melhoria da qualidade, o compromisso da alta direção é um fator crítico de sucesso. Para a consultora da qualidade do Hospital H1: "[...] o reconhecimento da importância da acreditação pela alta direção foi crucial durante a busca dos diferentes niveis de certificação que passamos".

Os enfermeiros assumem uma posição estratégica na implantação da acreditação devido a sua proximidade com os clientes, ao compromisso institucional que manifestam e a suas relações com outros profissionais de saúde. Na pesquisa, os profissionais de enfermagem apresentaram diferentes graus de envolvimento. Nos hospitais $\mathrm{H} 4$ e H5, o envolvimento desta categoria foi considerado apenas regular. Para o gerente de qualidade do Hospital H5:

[...] a acreditação traz novas tarefas para a enfermagem, tais como trabalhar com dados, indicadores e padrões, o que pode acarretar em sobrecarga de trabalho. Consequentemente, exige-se

Quadro 5. Impactos da acreditação - Impactos na satisfação de pacientes e no reconhecimento público.

\begin{tabular}{|l|c|c|c|c|c|c|c|c|}
\hline Impactos - satisfação dos pacientes e reconhecimento público & H1 & H2 & H3 & H4 & H5 & H6 & Média & $\begin{array}{c}\text { Desvio } \\
\text { padrão }\end{array}$ \\
\hline Satisfação dos pacientes/clientes com o serviço & 4 & 4 & 4 & 4 & 4 & 4 & $\mathbf{4 , 0 0}$ & $\mathbf{0 , 0 0}$ \\
\hline Reconhecimento público & 4 & 4 & 5 & 4 & 4 & 4 & $\mathbf{4 , 1 7}$ & $\mathbf{0 , 4 1}$ \\
\hline
\end{tabular}

Quadro 6. Graus de envolvimento dos segmentos funcionais com o processo de acreditação.

\begin{tabular}{|l|c|c|c|c|c|c|}
\hline \multirow{2}{*}{ Categorias Profissionais } & \multicolumn{5}{|c|}{ GRAUS DE ENVOLVIMENTO COM A ACREDITAÇ̃̃O } \\
\cline { 2 - 7 } & H1 & H2 & H3 & H4 & H5 & H6 \\
\hline Alta Direção & Alto & Alto & Alto & Alto & Alto & Alto \\
\hline Corpo de Enfermagem & Alto & Alto & Alto & Regular & Regular & Alto \\
\hline Corpo Médico & Regular & Regular & Regular & Regular & Baixo & Regular \\
\hline Corpo Administrativo & Alto & Alto & Regular & Regular & Baixo & Alto \\
\hline
\end{tabular}


mais tempo para que essa mudança de cultura seja consolidada no hospital.

O destaque negativo coube ao comprometimento do corpo médico, conforme já identificado na bibliografia. Para a maioria dos hospitais, este envolvimento foi considerado apenas regular, enquanto que para o Hospital H5, ele foi considerado baixo. Tal situação é inadequada, já que o médico deveria assumir um papel de liderança na acreditação, pois sua atuação influencia a percepção de qualidade do paciente, bem como o comportamento dos demais profissionais de saúde. Já o envolvimento do corpo administrativo foi avaliado diferentemente pelos entrevistados. As avaliações negativas couberam aos Hospitais H3, H4 e H5. Para esses hospitais, foi difícil envolver o corpo administrativo no processo de acreditação por serem mais resistentes às mudanças.

O Quadro 4 apresenta a percepção dos entrevistados em outros dois temas: desenvolvimento profissional e atitudes.

Quanto à relação entre acreditação e desenvolvimento profissional, tem-se que ela foi considerada mais positiva para os profissionais de enfermagem. A acreditação teria o potencial para desenvolver a liderança, estimular a formação de competências (interpessoais e gerenciais) e dar uma conotação mais gerencial à execução das rotinas desempenhadas pelo enfermeiro. Segue:o

[...] A enfermagem como sempre é o maior envolvido, pois a base é a segurança dos nossos pacientes/ clientelfamiliares. O apoio do grupo de enfermagem foi importantíssimo para desenvolver os protocolos e as atividades necessárias. Os demais grupos da instituição também tiveram seu peso na participação de implantações. Não dá para trabalhar sozinho, um setor sempre vai depender do outro, mais do que nunca o trabalho em equipe é essencial. (Hospital H2)

Em relação à influência da acreditação no desenvolvimento do corpo médico, os entrevistados dos hospitais H1, H2 e H3 anotaram avaliações positivas (Quadro 4). Já os entrevistados dos outros três hospitais (H4, H5 e H6) assinalaram avaliações neutras e negativas, denotando um compromisso moderado. Os trechos abaixo justificam a avaliação negativa:

[...] os médicos participam parcialmente, mas estamos trabalhando com frequência na tentativa de incluir o corpo médico na Gestão da Qualidade. (Hospital H4)

[...] pouco envolvimento e dificuldade de enquadramentos dos médicos, por se tratar de corpo clínico aberto. Neste caso, o engajamento tende a ser menor pois eles não se sentem pertencentes ao hospital. (Hospital H5)

[...] o corpo médico precisa ter um envolvimento maior, pois se não for bem explicado, eles podem dificultar a implantação. Para muitos, é um processo burocrático e há resistência em seguir os padrões adotados. (Hospital H6)

Como observado no Quadro 4, existe a percepção de que a acreditação gera uma atitude positiva nos profissionais de saúde, ainda que com as limitações apontadas acima. A supervisora de enfermagem do hospital H2 afirma: "estou muito entusiasmada com as mudanças que estão acontecendo, sinto que todos nós, tanto pacientes como funcionários, sairemos beneficiados de alguma forma".

\subsubsection{Impactos na satisfação de pacientes e no reconhecimento público}

O Quadro 5 apresenta a percepção dos entrevistados em relação aos impactos na satisfação dos pacientes e no reconhecimento público dos hospitais.

A relação entre acreditação e satisfação dos clientes também foi investigada. Dois fatores abordavam esta questão: segurança e diminuição de riscos aos pacientes (Quadro 3) e satisfação dos clientes/pacientes com os serviços prestados (Quadro 5) Os resultados denotam que a acreditação influencia positivamente a segurança dos pacientes e contribui para o aumento dos níveis de satisfação dos clientes/pacientes, conforme pode ser observado nos trechos abaixo:

[...] houve alterações positivas no aspecto de retorno ao paciente referente aos apontamentos nas pesquisas que realizamos. (Hospital H2)

[...] a pesquisa da satisfação que implementamos revela que hoje temos o paciente mais satisfeito $e$, com isso, ganhamos a sua fidelidade. (Hospital H3)

[...] a expectativa do cliente é difícil de ser trabalhada no hospital. Mesmo assim, o hospital busca a qualidade e melhoria da saúde do paciente. (Hospital H4)

Se o retorno financeiro pode ser de difícil mensuração, existe a percepção de que a acreditação tem impactos positivos no fortalecimento da imagem do hospital, conforme pode ser observado nos trechos abaixo:

[...] O reconhecimento já se tornou público, estamos no ranking dos hospitais notáveis da secretaria da saúde. (Hospital H3)

[...] A acreditação trouxe maior credibilidade junto às operadoras de planos de saúde e sociedade em geral. Temos uma excelente imagem em nossa região. (Hospital H5)

\subsection{Dificuldades no processo de implantação da acreditação}

A pesquisa também buscou identificar as dificuldades enfrentadas pelos hospitais durante o processo de acreditação. A dificuldade mais citada foi a de se mudar a cultura da organização, apontada por cinco 
hospitais (hospitais H1, H2, H3, H4 e H6). Como qualquer programa da qualidade, a acreditação exige mudanças de valores, hábitos e comportamentos das pessoas envolvidas. Fazer com que os profissionais de saúde e administrativo sigam os procedimentos estabelecidos, respeitem as regras de segurança e tenham a qualidade assistencial como valor essencial é fundamental para o sucesso da acreditação hospitalar. Por exemplo, o hospital H1 enfrentou muita resistência na notificação dos eventos sentinelas. Enquanto tais comportamentos não estiverem incorporados ao conjunto de crenças e valores da organização, não se conseguirá consolidar uma cultura orientada para a qualidade. Por isso, um grande esforço deve ser feito para que se possam minimizar eventuais resistências.

Outra dificuldade refere-se ao não envolvimento de algumas categorias profissionais, principalmente durante o processo de capacitação (citada pelos hospitais $\mathrm{H} 1, \mathrm{H} 2$ e H4), o que está relacionada também à questão cultural. $\mathrm{O}$ gerente da qualidade do Hospital H5 mencionou que o processo de acreditação acarretou sobrecarga de trabalho nos gestores, gerando estresses e cobranças advindos das demandas impostas. Apenas o Hospital H3 apontou dificuldades financeiras como relevantes ao processo de acreditação. Alguns trechos ilustram as dificuldades apontadas pelos entrevistados:

\section{[...] A principal como já mencionado é a mudança de cultura das equipes o que trabalhamos muito através de palestras e implantações de melhorias. (Hospital H2)}

[...] As dificuldades que mais impactam são as necessidades de recursos financeiros para adequação da estrutura fisica e de equipamentos médicos, que fogem ao custeio, não havendo verba especifica ... (Hospital H3)

[...] uma dificuldade para nós da equipe que coordena os trabalhos de acreditação é a falta de tempo e a sobrecarga no trabalho no acompanhamento dos processos, indicadores e realização de treinamentos. Isto gera uma sobrecarga, mas sabemos que o trabalho é importante. (Hospital H5)

Para os entrevistados, a superação das dificuldades identificadas passa pelo compromisso da alta direção com a acreditação e pela promoção de atividades de sensibilização e treinamento dos profissionais de saúde, conforme observa a consultora interna da qualidade do hospital H1:

[...] Buscamos superar as dificuldades com a conscientização da equipe por meio de treinamentos com foco na ação educativa e não punitiva. Para o envolvimento de algumas áreas realizamos reuniões com os coordenadores das áreas, explicando-lhes a importância da melhoria. Entretanto, tudo isto só é possivel com o apoio da direção do hospital, o principal exemplo deve ser deles.

\section{Implicações gerenciais}

Este trabalho traz implicações gerenciais. A primeira refere-se aos "retornos" da acreditação. A investigação sobre os impactos da acreditação é uma questão importante devido à escassez de pesquisas no cenário nacional. Em consonância com a bibliografia, os resultados deste estudo ratificaram que a acreditação é capaz de gerar mudanças organizacionais (Greenfield et al., 2012a; Alkhenizan \& Shaw, 2011). Entre essas, foram destacadas no estudo: a melhoria da organização interna por meio da gestão de processos (Pomey et al., 2010), a melhoria da qualidade assistencial (Al Tehewy et al., 2009) e o reconhecimento público (Greenfield \& Braithwaite, 2008). Entretanto, tais benefícios não têm sido suficientes para gerar uma adesão mais significativa dos hospitais brasileiros ao programa de acreditação.

Para a intensificação da acreditação como ferramenta de melhoria da qualidade hospitalar, seria importante a adoção, por parte das autoridades competentes, de estratégias que tornassem a acreditação mais atraente aos hospitais. Por exemplo, fazendo com que as principais fontes pagadoras dos serviços hospitalares (planos de saúde e o Estado) passassem a vincular a acreditação como condição de credenciamento ou remuneração dos serviços de saúde. Outra estratégia seria estimular o uso de práticas para "quantificar" economicamente os ganhos dos hospitais com a acreditação. Tais medidas foram adotadas com sucesso em outros países.

A segunda implicação refere-se à necessidade da mudança cultural imposta pela acreditação hospitalar. Como evidenciado nos resultados, um desafio para um hospital que busca a acreditação é conseguir o engajamento dos profissionais de saúde. Neste sentido, o comprometimento da alta direção torna-se imprescindível, cabendo a ela dar o exemplo a ser seguido pelas demais categorias profissionais (Macinati; 2008). Para Bergamini (1994), a liderança atribui significados às ações desenvolvidas pelos liderados, atuando como um agente de transformação.

No hospital, coexistem diferentes categorias profissionais de saúde que formam subculturas. Essa diversidade de grupos impõe desafios à implantação da acreditação. Enquanto os profissionais de enfermagem mostram possuir vínculos comprometidos com a acreditação, a categoria médica, dada a sua formação especialista e a precariedade de vínculos de contratação (em muitos hospitais), mostra-se menos comprometida. Assim, cabe à liderança reconhecer essa diversidade de interesses e descobrir maneiras de levar os médicos a participarem mais ativamente do processo de acreditação. Muitas vezes será preciso que o hospital repense seus processos de contratação, remuneração e avaliação de desempenho. Todavia, é importante que não só os hospitais, mas também os próprios organismos de acreditação desenvolvam estratégias para aumentar o engajamento médico. 


\section{Considerações finais}

O trabalho contribui ao ampliar os estudos sobre acreditação no Brasil. Para o setor hospitalar, cujo cenário revela tendências de modernização, de aumento da concorrência e de maior exigência dos clientes, a pesquisa se mostra relevante por buscar conhecer as práticas e impactos da acreditação nos hospitais estudados. Para cumprir este objetivo foi feito um levantamento da literatura existente sobre o tema. Adicionalmente, foram realizados casos em seis hospitais acreditados.

Os resultados mostraram que a acreditação hospitalar tem capacidade de gerar melhorias organizacionais quanto à gestão de processos, ao aumento da segurança aos pacientes, ao desenvolvimento dos profissionais de saúde e contribui também para a melhoria de imagem pública dos hospitais acreditados. Entretanto, a capacidade de a acreditação gerar benefícios financeiros aos hospitais pesquisados não pode ser evidenciada.

Um aprofundamento do estudo sobre os impactos da acreditação é essencial para se garantir mais adesões ao Programa Brasileiro de Acreditação. A segunda conclusão é que a acreditação, como qualquer iniciativa de qualidade, é um processo de mudança cultural, o qual deve ser cuidadosamente gerenciado pela liderança dos hospitais. Compromissos devem ser firmados para se garantir o envolvimento de todas as subculturas existentes no hospital.

O estudo apresenta limitações. Uma primeira limitação pode ser atribuída ao perfil dos hospitais pesquisados, que se diferenciavam em termos do porte, natureza, nível de acreditação e tempo já dedicado à acreditação hospitalar. Tais elementos podem ter influenciado as percepções dos entrevistados. Uma segunda limitação refere-se ao estágio de evolução do processo de acreditação brasileiro, que ainda é recente em comparação ao de outros países como França e Estados Unidos. Assim, pode-se inferir que seria necessário mais tempo para se refletir sobre os reais impactos da acreditação no Brasil. Uma última limitação pode ser atribuída ao método (pesquisa qualitativa por meio de estudos de casos) adotado para esta pesquisa.

Espera-se, com o desenvolvimento do presente estudo, acrescentar evidências sobre os impactos da acreditação hospitalar e, assim, motivar e subsidiar o desenvolvimento de novas pesquisas sobre o tema.

\section{Referências}

Al Tehewy, M., Salem, B., Habil, I., \& El Okda, S. (2009). Evaluation of accreditation program in non-governmental organizations' health units in Egypt: short-term outcomes. International Journal for Quality in Health Care, 21(3), 183-189. http://dx.doi.org/10.1093/intqhe/mzp014. PMid:19439444.
Alavi, J., \& Mahamoud, M. Y. (2008). The role of quality improvement initiatives in ealthcare operational environments. International Journal of Health Care Quality Assurance, 21(2), 133-145. http://dx.doi. org/10.1108/09526860810859003. PMid:18578199.

Alkhenizan, A., \& Shaw, C. (2011). Impact of accreditation on the quality of healthcare services: a systematic review of the literature. Annals of Saudi Medicine, 31(4), 407-416. http://dx.doi.org/10.4103/0256-4947.83204. PMid:21808119.

Auras, S., \& Geraedts, M. (2010). Patient experience data in practice accreditation--an international comparison. International Journal for Quality in Health Care, 22(2), 132-139. http://dx.doi.org/10.1093/intqhe/mzq006. PMid:20144943.

Bardin, L. (1977). Análise de conteúdo. Lisboa: Edições 70.

Bergamini, C. W. (1994). Liderança: administração do sentido. Revista de Administração de Empresas, 34(3), 102-114.

Burmester, H., Pereira, J. C., \& Scarpi, M. J. (2007). Modelo de Gestão para organizações de Saúde. Revista de Administração em Saúde, 9(37), 125-132.

Chen, J., Rathore, S. S., Radford, M. J., \& Krumholz, H. M. (2003). JCAHO accreditation and quality of care for acute myocardial infarction. Health Affairs, 22(2), 243-254. http://dx.doi.org/10.1377/hlthaff.22.2.243. PMid:12674428.

Davis, M. V., Reed, J., Devlin, L. M., Michalak, C. L., Stevens, R., \& Baker, E. (2007). The NC accreditation learning collaborative: partners enhancing local health department accreditation. Journal of Public Health Management and Practice, 13(4), 422-426. http:// dx.doi.org/10.1097/01.PHH.0000278038.38894.41. PMid:17563633.

Devers, K. J., Pham, H. H., \& Liu, G. (2004). What is driving hospitals' patient-safety efforts? Health Affairs, 23(2), 103-115. http://dx.doi.org/10.1377/hlthaff.23.2.103. PMid:15046135.

Eisenhardt, K. M. (1989). Building theories from case study research. Academy of Management Review, 14(4), 532-550.

El-Jardali, F., Jamal, D., Dimassi, H., Ammar, W., \& Tchaghchaghian, V. (2008). The impact of hospital accreditation on quality of care: perception of Lebanese nurses. International Journal for Quality in Health Care, 20(5), 363-371. http://dx.doi.org/10.1093/intqhe/ mzn023. PMid:18596050.

Feldman, L. B., Gatto, M. A. F., \& Cunha, I. C. K. O. (2005). História da evolução da qualidade hospitalar: dos padrões a acreditação. Revista Acta Paulista de Enfermagem, 18(2), 213-219.

Figueiredo, K. F. (2012). Panorama de Acreditação Hospitalar no Brasil 2009-2011. Rio de Janeiro: Centro de Estudos em Gestão de Serviços de Saúde, Universidade Federal do Rio de Janeiro. Relatório de Pesquisa. 
Forgia, G. M., \& Couttolenc, B. F. (2008). Hospital performance in Brazil: the search for excellence. Washington: The World Bank.

Fortes, M. T., Mattos, R. A., \& Baptista, T. W. (2011). Accreditation or accreditations? A comparative study about accreditation in France, United Kingdom and Cataluña. Revista da Associação Médica Brasileira, 57(2), 239-246. http://dx.doi.org/10.1590/S010442302011000200025 . PMid:21537714.

Greenfield, D., \& Braithwaite, J. (2008). Health sector accreditation research: a systematic review. International Journal for Quality in Health Care, 20(3), 172-183. http:// dx.doi.org/10.1093/intqhe/mzn005. PMid:18339666.

Greenfield, D., Pawsey, M., \& Braithwaite, J. (2011). What motivates professionals to engage in the accreditation of healthcare organizations? International Journal for Quality in Health Care, 23(1), 8-14. http://dx.doi. org/10.1093/intqhe/mzq069. PMid:21084322.

Greenfield, D., Pawsey, M., \& Braithwaite, J. (2012a). The role and impact of accreditation on the healthcare revolution. Acreditação, 1(2), 1-14.

Greenfield, D., Pawsey, M., Hinchcliff, R., Moldovan, M., \& Braithwaite, J. (2012b). The standard of healthcare accreditation standards: a review of empirical research underpinning their development and impact. $B M C$ Health Services Research, 12(1), 329. http://dx.doi. org/10.1186/1472-6963-12-329. PMid:22995152.

Hasan, M., \& Kerr, R. M. (2003). The relationship between total quality management practices and organizational performance in service organizations. The TQM Magazine, 15(4), 286-291. http://dx.doi. org/10.1108/09544780310486191.

Hinchcliff, R., Greenfield, D., Moldovan, M., Westbrook, J. I., Pawsey, M., Mumford, V., \& Braithwaite, J. (2012). Narrative synthesis of health service accreditation literature. BMJ Quality \& Safety, 21(12), 979-991. http://dx.doi. org/10.1136/bmjqs-2012-000852. PMid:23038406.

Lee, S., Choi, K. S., Kang, H. Y., Cho, W., \& Chae, Y. M. (2002). Assessing the factors influencing continuous quality improvement implementation: experience in Korean hospitals. International Journal for Quality in Health Care, 14(5), 383-391. http://dx.doi.org/10.1093/ intqhe/14.5.383. PMid:12389804.

Macinati, M. S. (2008). The relationship between quality management systems and organizational performance in the Italian National Health Service. Health Policy, 85(2), 228-241. http://dx.doi.org/10.1016/j.healthpol.2007.07.013. PMid:17825941.

Malik, A. M., \& Teles, J. P. (2001). Hospitais e programas de qualidade no Estado de São Paulo. Revista de Administração Hospitalar, 41(3), 51-59.

Manaf, N. H. A. (2005). Quality management in Malaysian public health care. International Journal of Health Care Quality Assurance, 18(2-3), 204-216. http://dx.doi. org/10.1108/09526860510594767. PMid:15974516.
Manzo, B. F. (2009). O processo de Acreditação Hospitalar na perspectiva de profissionais de saúde (Dissertação de mestrado). Escola de Enfermagem, Universidade Federal de Minas Gerais, Belo Horizonte.

Manzo, B. F., Ribeiro, H. C. T. C., Brito, M. J. M., \& Alves, M. (2012). A enfermagem no processo de acreditação hospitalar: atuação e implicações no cotidiano de trabalho. Revista Latino-Americana de Enfermagem, 20(1), 151-158. http://dx.doi.org/10.1590/S010411692012000100020 . PMid:22481733.

Mezomo, J. C. (2001). Gestão da qualidade na saúde: princípios básicos (1 ed.). São Paulo: Loyola. 301 p.

Miller, M. R., Pronovost, P., Donithan, M., Zeger, S., Zhan, C., Morlock, L., \& Meyer, G. S. (2005). Relationship between performance measurement and accreditation: implications for quality of care and patient safety. American Journal of Medical Quality, 20(5), 239252. http://dx.doi.org/10.1177/1062860605277076. PMid:16221832.

Mumford, V., Forde, K., Greenfield, D., Hinchcliff, R., \& Braithwaite, J. (2013). Health services accreditation: what is the evidence that the benefits justify the costs? International Journal for Quality in Health Care, 25(5), 606-620. http://dx.doi.org/10.1093/intqhe/mzt059. PMid:23942825.

Newhouse, R. P. (2006). Selecting measures for safety and quality improvement initiatives. The Journal of Nursing Administration, 36(3), 109-113. http://dx.doi. org/10.1097/00005110-200603000-00002. PMid:16601511.

Olástico, G. P., \& Toledo, J. C. (2013). Acreditação Hospitalar: proposição de roteiro para implantação. Gestão \& Produção, 20(4), 815-831. http://dx.doi. org/10.1590/S0104-530X2013005000011.

Organização Nacional de Acreditação - ONA. (2013). Manual das organizações prestadoras de serviços hospitalares. Recuperado em 1 de dezembro de 2013, de http://www.ona.org.br

Ovretveit, J. \& Gustafson, D. (2002). Evaluation of quality improvement programmes. Quality Safety Care, 11, 270-275

Ovretveit, J. (1996). Medical participation in and leadership of quality programmes. Journal of Management in Medicine, 10(5), 21-28. http://dx.doi.org/10.1108/02689239610146526. PMid:10166029.

Pomey, M. P., Contandriopoulos, A. P., François, P., \& Bertrand, D. (2004). Accreditation: a tool for organizational change in hospitals? International Journal for Quality in Health Care, 17(2-3), 113-124. http://dx.doi. org/10.1108/09526860410532757. PMid:15301268.

Pomey, M. P., François, P., Contandriopoulos, A. P., Tosh, A., \& Bertrand, D. (2005). Paradoxes of French accreditation. Quality \& Safety in Health Care, 14(1), 51-55. http://dx.doi.org/10.1136/qshc.2004.011510. PMid:15692004.

Pomey, M. P., Lemieux-Charles, L., Champagne, F., Angus, D., Shabah, A., \& Contandriopoulos, A. P. (2010). Does 
accreditation stimulate change? A study of the impact of the accreditation process on Canadian healthcare organizations. Implementation Science, 5(31), 31. http:// dx.doi.org/10.1186/1748-5908-5-31. PMid:20420685.

Sack, C., Scherag, A., Lütkes, P., Günther, W., Jöckel, K. H., \& Holtmann, G. (2011). Is there an association between hospital accreditation and patient satisfaction with hospital care? A survey of 37,000 patients treated by 73 hospitals. International Journal for Quality in Health Care, 23(3), 278-283. http://dx.doi.org/10.1093/ intqhe/mzr011. PMid:21515636.

Sekimoto, M., Imanaka, Y., Kobayashi, H., Okubo, T., Kizu, J., Kobuse, H., Mihara, H., Tsuji, N., \& Yamaguchi, A., \& Japan Council for Quality Health Care, Expert Group on Healthcare-Associated Infection Control and Prevention (2008). Impact of hospital accreditation on infection control programs in teaching hospitals in Japan. American Journal of Infection Control, 36(3), 212-219. http://dx.doi.org/10.1016/j.ajic.2007.04.276. PMid:18371518.

Shaw, C., Groene, O., Moura, N. \& Sunol, R. (2010). Accreditation and ISO certification: do they explain differences in quality management in European hospitals? International Journal for Quality in Health Care, 22(6), 445-451. http://dx.doi.org/10.1093/intqhe/mzq054.

Shiesari, L. M. C., \& Kisil, M. (2003). A avaliação da qualidade nos hospitais brasileiros. Revista de Administração em Saúde, 5(18), 7-17.

Tabrizi, J. S., Gharibi, F., \& Wilson, A. J. (2011). Advantages and disadvantages of health care accreditation mod-els. Health Promotion Perspectives, 1(1), 1-31. PMid:24688896.

Thornlow, D. K., \& Merwin, E. (2009). Managing to improve quality: the relationship between accreditation standards, safety practices, and patient outcomes. Health Care Management Review, 34(3), 262-272. http://dx.doi. org/10.1097/HMR.0b013e3181a16bce. PMid:19625831.

Touati, N., \& Pomey, M. P. (2009). Accreditation at a crossroads: are we on the right track? Health Policy, 90(2-3), 156-165. http://dx.doi.org/10.1016/j. healthpol.2008.09.007. PMid:18995923.

Yin, R. K. (2001). Estudo de caso: planejamento e métodos. Porto Alegre: Bookman. 